\title{
SENP2 exerts an anti-tumor effect on chronic lymphocytic leukemia cells through the inhibition of the Notch and NF- $\kappa B$ signaling pathways
}

\author{
XIU-LI CHEN $^{1,2^{*}}$, SHI-FEN WANG ${ }^{1 *}$, XUE-TING LIANG $^{1}$, HUI-XIN LIANG ${ }^{1}$, TING-TING WANG ${ }^{1}$, \\ SHUN-QUAN WU ${ }^{1}$, ZONG-JIAN QIU ${ }^{1}$, RONG ZHAN ${ }^{1}$ and ZHEN-SHU XU ${ }^{1}$ \\ ${ }^{1}$ Fujian Institute of Hematology, Fujian Provincial Key Laboratory on Hematology, \\ Fujian Medical University Union Hospital, Fuzhou, Fujian 350001; ${ }^{2}$ Department of Hematology, \\ The First Hospital of Putian City, Putian, Fujian 351100, P.R. China
}

Received June 20, 2018; Accepted October 19, 2018

DOI: 10.3892/ijo.2018.4635

\begin{abstract}
Chronic lymphocytic leukemia (CLL) is one of the most often diagnosed hematological malignant tumors in the Western world and a type of inert B-cell lymphoma that commonly attacks the elderly. Small ubiquitin related modifier (SUMO)-specific protease 2 (SENP2) can act as a suppressor in various types of cancer by regulating the stability of $\beta$-catenin to affect the Notch signaling pathway; however, it has a low expression level in CLL cells. In this study, we firstly used western blot analysis and RT-qPCR to detect the protein and mRNA expression levels of SENP2 in the peripheral blood of patients with CLL and healthy volunteers. Secondly, we overexpressed or knocked down the expression of SENP2 in CLL cells and then determined the cell invasive and chemotactic ability in a Transwell assay and chemotaxis assay. We examined the sensitivity of the cells to cytarabine and dexamethasone via a CCK- 8 assay and determined the cell apoptotic condition and the expression of the Notch signaling pathway using flow cytometry and western blot analysis. The results demonstrated that the patients with CLL had relatively low expression levels of SENP2. The overexpression of SENP2 in the CLL cells decreased their invasive and proliferative ability, as well as their chemotactic response and enhanced their sensitivity to cytarabine and dexamethasone, while it promoted cell apoptosis. The silencing of SENP2 in the CLL cells generally
\end{abstract}

Correspondence to: Professor Zhen-Shu Xu, Fujian Institute of Hematology, Fujian Provincial Key Laboratory on Hematology, Fujian Medical University Union Hospital, 29 Xinquan Road, Fuzhou, Fujian 350001, P.R. China

E-mail: zhenshuxu@yahoo.com

${ }^{*}$ Contributed equally

Key words: chronic lymphocytic leukemia, small ubiquitin related modifier (SUMO)-specific protease 2, $\beta$-catenin, Notch signaling pathway produced the opposite results. We thus hypothesized that the overexpression of SENP2 downregulated $\beta$-catenin expression, thus inhibiting the Notch signaling pathway in CLL cells. Moreover, the nuclear factor (NF) $-\kappa \mathrm{B}$ signaling pathway was also regulated by the overexpression of SENP2. On the whole, the findings of this study indicate tha SENP2 can act as a tumor suppressor in CLL cells, and may thus prove to be a novel target for CLL treatment in clinical practice.

\section{Introduction}

Chronic lymphocytic leukemia (CLL) is the most frequent and wide-spread type of leukemia affecting adults (1). Each year, 15,000 new patients are diagnosed with CLL, which is approximately $1.1 \%$ of the cancer-related incidence, and approximately 5,000 patients succumb to the disease, which is approximately $0.8 \%$ of cancer-associated mortality in the United States (1). CLL has a higher probability of occurring in the elderly. The majority of patients with CLL are 65 years old or older, and the incidence and mortality of CLL in China has gradually increased in recent years. This could be attributed to the huge population base and an aging society (2). As a type of slow-growing B-cell lymphoma, CLL usually begins with the aggregation of large numbers of malignant and mature B-lymphocytes in the peripheral blood, bone marrow and secondary lymphoid tissues. This subsequently leads to a disorder in or to the collapse of hematopoietic function (1). However, the diagnosis of CLL always occurs in the early-intermediate stages of the disease and $90 \%$ of the patients are asymptomatic $(3,4)$. Moreover, there is no clinical evidence that early therapy would improve the survival rate of patients with CLL. Actually, many patients with CLL exhibit indolent symptoms and have a good life expectancy, whereas minorities face the rapid development of disease and a poor prognosis (3). Currently, the preferred treatment for CLL is chemoimmunotherapy; however, the pharmaceutical effects do not achieve the desired efficiency, which may be blocked by the still unclear pathogenesis of CLL (5). Therefore, it is of utmost importance to intensively explore the molecular mechanisms responsible for CLL initiation and development 
and to identify novel targets for the precise diagnosis and treatment of CLL.

Small ubiquitin related modifier (SUMO)-specific protease 2 (SENP2) is a member of the SUMO-specific protease (SENP) family that is essential to the processes of SUMOylation and de-SUMOylaton $(6,7)$. Among the three subfamilies of SENPs, SENP2 can be classified as a member of the first subfamily, which is characterized by broad substrate specificity (8). SENP2 is localized in the nuclear pore through binding with the Nup153 nucleoporin and a nuclear envelope-related protease. It has been demonstrated that SENP2 plays a critical role in embryonic erythropoiesis when overexpressed, which is similar to SENP1 (9). Previously, some researchers reported that SENP2can promote the development of embryonic erythropoiesis by regulating the $\mathrm{p} 53-\mathrm{Mdm} 2$ signaling pathway in the placenta $(10,11)$. However, it has also been reported that SENP2 plays a potential role in the tumorigenesis of various types of cancer, such as hepatocellular carcinoma, breast cancer and bladder cancer (12-15). For example, the study by Shen et al reported that the overexpression of SENP2 in hepatocellular carcinoma cells inhibited cell proliferation through the regulation of $\beta$-catenin stability, while the opposite effect was observed by the silencing of SENP2 (14). Moreover, the study by Tan et al also illustrated the downregulation of SENP2 in bladder cancer tissues and the inhibition of the migratory and invasive ability of bladder cancer cells by the overexpression of SENP2 through the blocking if the activation of matrix metalloproteinase (MMP)13 in vitro (13). The study by Nait Achour et al verified that SENP2 suppressed the proliferation of estrogen-dependent or-independent MCF7 breast cancer cells by preventing the interaction between the SENP2 and ER $\alpha$ proteins (12). However, whether SENP2 is involved in the development and occurrence of CLL has not been extensively explored and warrants further investigation.

The Notch signaling pathway plays important roles in the proliferation, differentiation, apoptosis, and other physiological activities of normal cells and has been identified as an evolutionarily conserved signaling pathway (16). However, the abnormal activation of the Notch signaling pathway in CLL has also been reported by a number of studies and the overexpression and mutation of some Notch molecules has been reported to be associated with drug resistance, a poor prognosis, and other issues in CLL (17-23). Nwabo Kamdje et al and Rosati et al found that some Notch receptors such as Notchl and Notch2, and ligands such as Jaggedl and Jagged2 have a high expression in patients with CLL and in primary CLL cells $(17,18)$. In addition, the activation of the Notch signaling pathway is associated with the nuclear factor $(\mathrm{NF})-\kappa \mathrm{B}$ signaling pathway and NF- $\mathrm{B}$ can upregulate the expression of Jagged1, which interacts with Notch to continually activate the Notch signaling pathway in CLL cells $(24,25)$. Notably, Sun et al identified $\mathrm{Wnt} / \beta$-catenin signaling as the signaling pathway downstream of Notch and the mechanism of the promoting effect of hepatocarcinogenesis by Notch1 (26). Jiang et al also reported that SENP2 inhibited the growth of hepatocellular carcinoma cells by the modulation of $\beta$-catenin stability through WW domain-containing oxidoreductase (WWOX), a novel inhibitor of the Wnt/ $\beta$-catenin pathway (15). Therefore, we inferred that SENP2 may also inhibit the occurrence and development of CLL via the regulation of $\beta$-catenin to affect the Notch signaling pathway.

In this study, we first detected the protein and mRNA expression levels of SENP2 in patients with CLL. We then established CLL cells in which SENP2 was overexpressed or silenced to determine their invasive and chemotactic ability, their sensitivity to cytarabine and dexamethasone, the cell apoptotic state, the expression level of $\beta$-catenin, the activation state of the Notch and NF- $\mathrm{BB}$ signaling pathways, and other processes. This study aimed to clearly determine whether SENP2 functions as a tumor suppressor in CLL through the modulation of the Notch and NF- $\kappa \mathrm{B}$ signaling pathways.

\section{Materials and methods}

Samples, cells, antibodies and reagents. Peripheral blood from 43 patients with CLL (26/43 before treatment and 17/43 post-treatment; 15 female and 28 male patients; age range, 47-80 years) and 21 healthy volunteers ( 8 female and 13 male healthy volunteers; age range, 50-74 years) was collected (January, 2016 to July, 2017) at the Fujian Medical University Union Hospital (Fuzhou, China). Sex, age, stage and the expression level of serum lactate dehydrogenase ( $\mathrm{LDH}), \beta 2$-microglobulin ( $\beta 2-\mathrm{MG})$, and cell genetics of the CLL patients were also collected from the Fujian Medical University Union Hospital (Fuzhou, China). The study was approved by the Ethics Committee of the Fujian Medical University Union Hospital and written consent was obtained from all participants in this study.

The human CLL cell line, MEC2, was purchased from the American Type Culture Collection (ATCC, Manassas, VA, USA). All cells were maintained in RPMI-1640 medium supplemented with $10 \%$ fetal bovine serum (FBS) (both from Gibco/Thermo Fisher Scientific, Waltham, MA, USA), at $37^{\circ} \mathrm{C}$ in an atmosphere containing $5 \% \mathrm{CO}_{2}$ in $10 \mathrm{~cm}^{2}$ plates at $0.3-0.5 \times 10^{6}$ cells $/ \mathrm{ml}$. The primary antibodies used for western blot analysis and flow cytometry were purchased from various companies. The anti-Bcl-2 (1:1,000; cat. no. 2870), anti-Bax (1:1,000; cat. no. 5023), anti-cleaved caspase-9 (1:1,000; cat. no. 7237), anti-caspase-9 (1:1,000; cat. no.9508), anti-cleaved caspase-3 (1:1,000; cat. no. 9664), anti-caspase-3 (1:1,000; cat. no. 9665), anti-Cyld (1:1,000; cat. no. 12797), anti-c-Myc (1:1,000; cat. no. 5605), anti-Notch1 (1:3,000; cat. no. 3268), anti- $\beta$-catenin $(1: 1,000$; cat. no. 8480$)$, anti-IKK $\beta(1: 1,000$; cat. no. 8943), anti-IкB $\alpha$ (1:1,000; cat. no. 4812), anti-p65 (1:1,000; cat. no. 8242), anti-p50 (1:1,000; cat. no. 12540) and anti-p53 (1:1,000; cat. no. 2524) antibodies were from Cell Signaling Technology (Danvers, MA, USA). The anti-IKK $\alpha$ (1:1,000; cat. no. ab32041) and anti-GAPDH $(1: 1,000$; cat. no. ab37168) antibodies were from Abcam (Cambridge, UK). The anti-SENP2 (1:5,000; cat. no. sc-46638) antibody was obtained from Santa Cruz Biotechnology (Santa Cruz, CA, USA). The Cell Counting kit-8 was from Dojindo Laboratories (Kumamoto, Japan). Cytarabine and dexamethasone were purchased from Selleck Chemicals (Houston, TX, USA).

Establishment of CLL cells in which SENP2 was overexpressed or silenced. The primer for SENP2 was designed using Primer 5.0 software and provided by Sangon Biotech (Shanghai) Co., Ltd. (Shanghai, China). The cDNA sequence 
of SENP2 was amplified from pGEM-SENP2 and constructed into the vector pBABE-hygro to generate pBABE-SENP2 by BioSCIRes Biotech Co., Ltd. (Shanghai, China). The vector $\mathrm{pBABE}-\mathrm{SENP} 2$ was used to prepare retroviral preparations for co-transfection of 293T cells (ATCC) with liposomes. Subsequently, shRNA sequences targeting SENP2 were designed by RNAi designer, and synthesized by Sangon Biotech (Shanghai) Co., Ltd. The shRNA sequences were inserted into the PLVX vector to generate PLVX-shRNA-SENP2 BioSCIRes Biotech Co., Ltd.). The mixture of PLVX-shRNA-SENP2, psPAX2 and pMDG2 was transfected into 293 T cells using Lipofectamine 2000 reagent (Invitrogen/Thermo Fisher Scientific) to generate lentivirus. Eventually, the MEC 2 cells, at a density of $8 \times 10^{4}$ cells $/ \mathrm{ml}$, were infected with the recombinant of lentivirus plus $8 \mu \mathrm{g} / \mathrm{ml}$ polybrene (Sigma, St. Louis, MO, USA).

Western blot analysis. The CLL cells in which SENP2 was overexpressed or silenced were seeded in 6-cm dishes. After reaching $70 \%$ confluence, cytarabine $(0.128 \mu \mathrm{g} / \mathrm{ml})$ or dexamethasone $(630 \mu \mathrm{g} / \mathrm{ml})$ or DMSO were added and the cells were further incubated for $24 \mathrm{~h}$ in a humidified atmosphere at $37^{\circ} \mathrm{C}$ with $5 \% \mathrm{CO}_{2}$. Subsequently, all CLL cells were collected by centrifugation $(1,000 \mathrm{rpm})$ at $4^{\circ} \mathrm{C}$ for $10 \mathrm{~min}$ and kept in RIPA lysis buffer (Beyotime, China) for $30 \mathrm{~min}$ at $4^{\circ} \mathrm{C}$ for cell lysis, then collected by centrifugation $(12,000 \mathrm{rpm})$ at $4^{\circ} \mathrm{C}$ for $15 \mathrm{~min}$. The supernatant was collected and the acquired total protein was measured using a BCA kit (Beyotime, China). Next, $20 \mu \mathrm{g}$ protein was separated by $10-12 \%$ SDS-PAGE and then transferred onto PVDF Transfer Membranes (Millipore, Billerica, MA, USA). Following the blocking of these membranes by $5 \%$ non-fat milk in TBST $(0.1 \%$ Tris-buffered saline with $1 \mathrm{ml} / 1$ Tween-20) for $1 \mathrm{~h}$ at room temperature, they were incubated with the corresponding primary antibodies at $4^{\circ} \mathrm{C}$ overnight. After washing with TBST 3 times, the membranes were further incubated with HRP-conjugated secondary antibodies (anti-goat, 1:2000, cat. no. A0181; anti-rabbit, 1:2000, cat. no. A0208; anti-mouse, 1:2000, cat. no. A0216) (all from Beyotime, China) for $1 \mathrm{~h}$ at room temperature. Finally, the bands were displayed by a SuperSignal West Pico Chemiluminescent Substrate (Thermo Fisher Scientific, Massachusetts, MA, USA) according to the protocol provided by the manufacturer. Western blot quantification was carried out using ImageJ software. Signals were adjusted for background and normalized to the signal for GAPDH.

Reverse transcription-quantitative PCR (RT-qPCR). In brief, we first designed the primers for SENP2 and GAPDH as shown in Table I; GAPDH was used as the reference gene. Second, peripheral blood lymphocytes (PBLCs) of patients with CLL were obtained using the lymphocyte isolation kit (cat. no. LTS1077; Tian Jin Hao Yang Biological Manufacture Co., Ltd., Tianjin, China). Total RNA was then extracted from the samples obtained from the PBLCs of patients with CLL according to the one-step TRIzol extraction. Third, the measurement of total RNA quality was conducted on a Nano-100 Micro Spectrophotometer (Hangzhou Allsheng Instruments Co., Ltd., Hangzhou, China) and the cDNA of SENP2 and GAPDH were then reverse transcribed through a general PCR step according to the PrimeScript RT reagent
Table I. The primer sequences of the related genes.

\begin{tabular}{lll}
\hline Gene & & Primer sequence (5'-3') \\
\hline SENP2 & Forward & CATTGGAGCCTGGTGGTGAT \\
SENP2 & Reverse & TGTTGAGGAATCTCGTGTGGTT \\
GAPDH & Forward & CGGATTTGGTCGTATTGGG \\
GAPDH & Reverse & GATTTTGGAGGGATCTCGC \\
\hline
\end{tabular}

kit (Takara Biomedical Technology Co., Ltd., Beijing, China). Finally, the levels of SENP2 and GAPDH cDNA were determined by qPCR according to the instructions provided with the SYBR Premix Ex Taq kit (Takara Biomedical Technology Co., Ltd, Beijing, China) to calculate the relative mRNA expression level of SENP2. The relative expression level of SENP2 was determined according to the $2^{-\Delta \Delta \mathrm{Cq}}$ method, as previously described (27).

Transwell assay. The Transwell system (24-wells, $8 \mu \mathrm{m}$ pore size with polycarbonate membrane; Corning Costar, Lowell, MA, USA) was used for the cell invasion assay. Briefly, $60 \mu \mathrm{l}$ of Matrigel were diluted by RPMI-1640 at a ratio of 1:7 then added into the upper wells of pre-cooled Transwell plates, and the plates were then incubated at $37^{\circ} \mathrm{C}$ for $4 \mathrm{~h}$. Subsequently, the CLL cells in which SENP2 was overexpressed or silenced were harvested and suspended in RPMI-1640 medium without FBS, and then $1 \times 10^{6}$ cells were added per well to the upper wells. Following incubation in a humidified atmosphere at $37^{\circ} \mathrm{C}$ with $5 \% \mathrm{CO}_{2}$ for $24 \mathrm{~h}$, the number of cells that crossed the Matrigel and moved into the lower wells were counted using a hemocytometer.

CCK- 8 assay. The CLL cells in which SENP2 was overexpressed or silenced were seeded in 96-well plates at a density of $2 \times 105$ cells $/ \mathrm{ml}$ and incubated with at $37^{\circ} \mathrm{C}$ for $48 \mathrm{~h}$. These cells were then incubated with concentration gradients of cytarabine or dexamethasone or DMSO from 0 to $10 \mathrm{mg} / \mathrm{ml}$ and for each concentration, there were 5 parallel control wells. The cells were then further incubated for 24,48 or $72 \mathrm{~h}$ before the CCK- 8 reagents $(10 \mu \mathrm{l})$ were added. All plates were shaken for a further $15 \mathrm{~min}$ on a shaker and the absorbance was measured at $450 \mathrm{~nm}$ using a Varioskan Flash multimode reader (Thermo Fisher Scientific).

Chemotaxis chamber assay. The chemotactic response of the CLL cells in which SENP2 was overexpressed or silenced was detected in96-well chemotaxis chambers with polycarbonate filters (5 $\mu \mathrm{m}$ pore size, $3.2 \mathrm{~mm}$ diameter size, $30 \mu \mathrm{l}$ well size) (Chemo TX Disposable Chemotaxis System, NeuroProbe, Gaithersburg, MD, USA). In brief, the CLL cells in which SENP2 was overexpressed or silenced were cultured under standard conditions and then suspended in RPMI-1640. The suspended CLL cells $\left(2 \times 10^{5}\right.$ cells $\left./ \mathrm{ml}\right)$ were added to the upper chamber. The dilution of CXCL12 (200 ng/ml) were added to the lower chamber and the cells were incubated for $8 \mathrm{~h}$ in a humidified atmosphere at $37^{\circ} \mathrm{C}$ with $5 \% \mathrm{CO}_{2}$. Subsequently, the CLL cells that did not migrate were removed from the membrane surface in the upper chamber using a cotton swab, and the CLL cells 

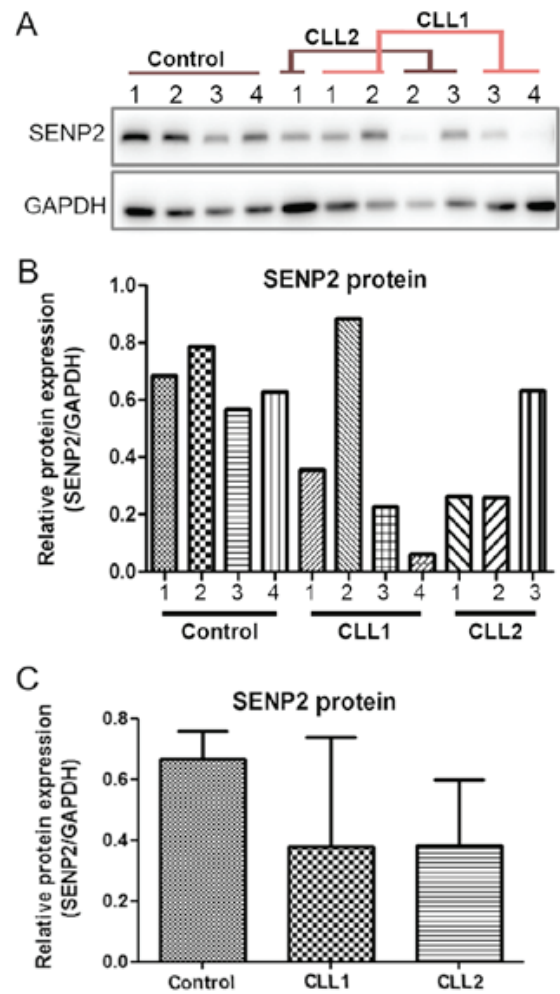

D

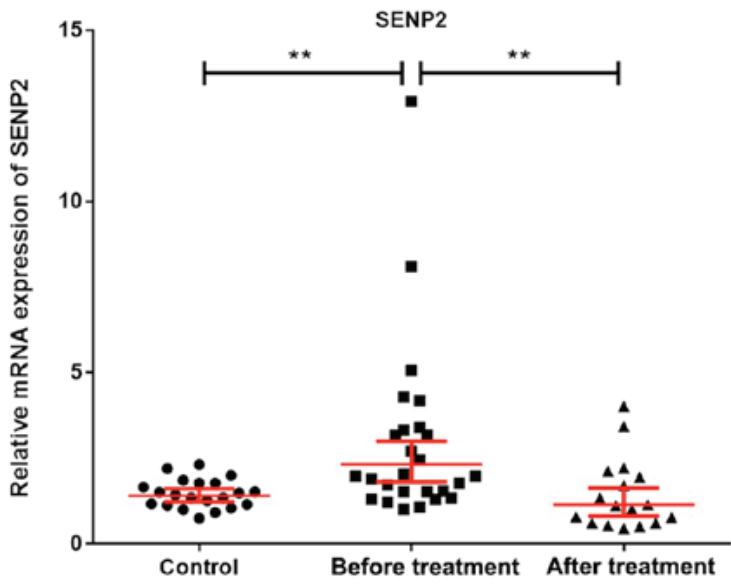

$\mathrm{E}$
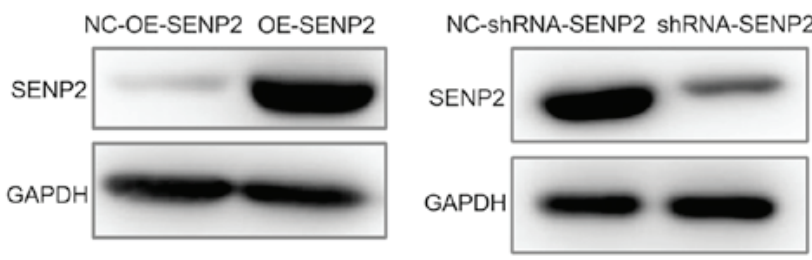

Figure 1. SENP2 is downregulated in the peripheral blood of patients with CLL. (A-C) The protein expression levels of SENP2 in the peripheral blood of patients with CLL and healthy volunteers detected by western blot analysis. (D) The mRNA expression levels of SENP2 in the peripheral blood of patients with CLL and healthy volunteers detected by RT-qPCR. Significant differences were detected between the 3 groups ("** $<0.01$ ), determined by the Kruskal-Wallis test. Significant differences were observed between the before treatment group with the control group and with the after treatment group separately (P=0.0034), determined by the Mann-Whitney test with the P-values and significance threshold corrected by the Bonferroni correction. (E) Western blot analysis was used to verify the successful construction of CLL cells in which SENP2 was overexpressed or silenced. CLL, chronic lymphocytic leukemia; CLL1, patients with CLL before treatment; CLL2, patients with CLL after treatment; NC-OE-SENP2, MEC2 cells transfected with null (control) overexpression vector; OE-SENP2, MEC2 cells transfected with SENP2 overexpression vector; NC-shRNA-SENP2, MEC2 cells transfected with null control shRNA; shRNA-SENP2, MEC2 cells transfected with shRNA against SENP2.

that had migrated to the lower surface of the membrane were fixed with 4\% paraformaldehyde (PFA) for $10 \mathrm{~min}$ and then labeled with DAPI (Sigma) to mark the cell nuclei. The number of migrating cells in 5 random fields was counted at x50 magnification by using a double-blinded approach, and images were captured at x200 magnification with a fluorescent microscope (LEICA DM 4000B; Leica, Wetzlar, Germany).

Flow cytometric analysis. The CLL cells in which SENP2 was overexpressed or silenced were seeded into $6 \mathrm{~cm}$ dishes $\left(1 \times 10^{5}\right.$ cells $\left./ \mathrm{ml}\right)$. Cytarabine $(0.128 \mu \mathrm{g} / \mathrm{ml})$ or dexamethasone $(630 \mu \mathrm{g} / \mathrm{ml})$ were used to treat the CLL cells that were further cultured for 24, 48 or $72 \mathrm{~h}$. Subsequently, an Annexin V-FITC Apoptosis Detection kit (BD Biosciences, San Jose, CA, USA) was used for apoptosis detection. In brief, the cells were harvested and suspended in binding buffer (100 $\mu \mathrm{l})$, achieving a final concentration of $1 \times 10^{6} / \mathrm{ml}$. Annexin V-FITC (5 $\left.\mu \mathrm{l}\right)$ and propidium iodide (PI, $20 \mu \mathrm{g} / \mathrm{ml}, 5 \mu \mathrm{l}$ ) were added and the cells were further cultured for $15 \mathrm{~min}$ at room temperature in the dark. The cells were analyzed by flow cytometry (FacsCalibur flow cytometer (BD Biosciences, Franklin Lakes, NJ, USA) following the addition of binding buffer $(400 \mu \mathrm{l})$. The obtained data were processed using FlowJo 7.6 software.

Statistical analysis. In this study, all data are expressed as the means $\pm \mathrm{SD}(n \geq 3)$ without special instructions. The
Kruskal-Wallis test was used for multiple group comparisons; the Mann-Whitney test was used for group comparisons with the P-values and significance threshold corrected by the Bonferroni correction. A paired-samples t-test or the Wilcoxon signed rank test were used for paired-samples analysis. Statistical analysis was carried out using SPSS15.0 software. Statistical differences with a value of $\mathrm{P}<0.05$ were considered significant.

\section{Results}

SENP2 is downregulated in the peripheral blood of patients with CLL. Peripheral blood was collected from 43 patients with CLL (26/43 before treatment and 17/43 post-treatment) and 21 healthy volunteers, and western blot analysis was used to detect the protein levels of SENP2 in PBLCs. The results of some randomly selected samples are shown in Fig. 1A-C. It was found that the protein level of SENP2 was generally lower in the peripheral blood of patients with CLL when compared with that of the healthy volunteers.

We further utilized RT-qPCR to detect the mRNA levels of SENP2 in peripheral blood mononuclear cells of 43 patients with CLL(26/43 before treatment and 17/43post R-FC chemotherapy treatment) and 21 healthy volunteers. As shown in Fig. 1D, patients with CLL without treatment exhibited higher mRNA levels of SENP2 than the healthy volunteers 

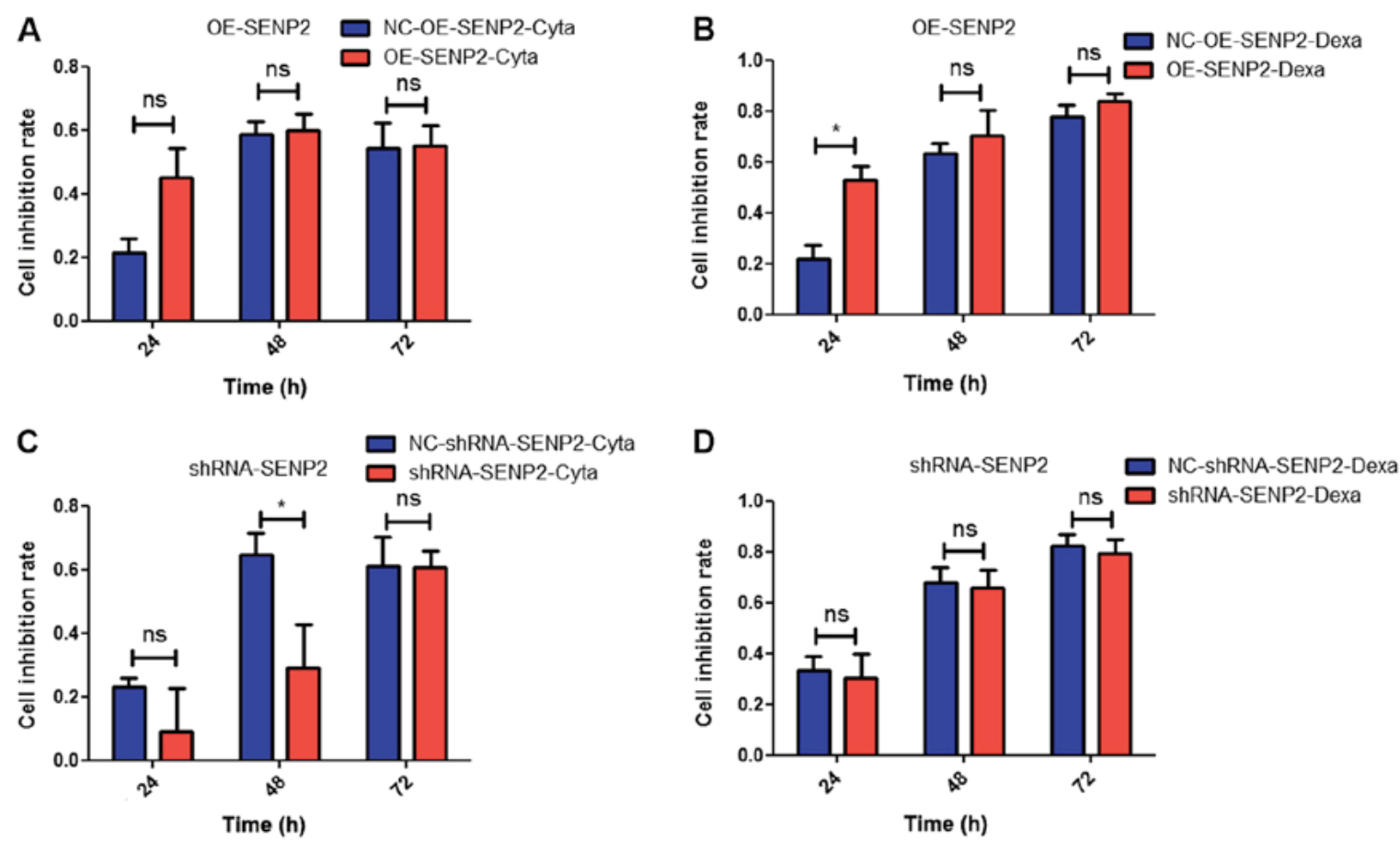

Figure 2. SENP2 has the tendency to enhance the sensitivity of CLL cells to cytarabine and dexamethasone. (A and B) MEC2CLL cells in which SENP2 was overexpressed cells and the control cells were treated as described in the Materials and methods. CCK- 8 assay was performed to examine the cell inhibition rate following treatment with (A) cytarabine and (B) dexamethasone. (C and D) MEC2 cells in which SENP2 was silenced and the control cells were treated as described in the Materials and methods and CCK-8 assay was performed to examine the cell inhibition rate following treatment with (C) cytarabine and (D) dexamethasone. The statistical method used is a paired-samples t-test. ${ }^{*} \mathrm{P}<0.05$; ns, no statistical significance; CLL, chronic lymphocytic leukemia; NC-OE-SENP2, MEC2 cells transfected with null (control) overexpression vector; OE-SENP2, MEC2 cells transfected with SENP2 overexpression vector; NC-shRNA-SENP2, MEC2 cells transfected with null control shRNA; shRNA-SENP2, MEC2 cells transfected with shRNA against SENP2; Cyta, cytarabine; Dexa, dexamethasone.

(control) group and the post-treatment group $(\mathrm{P}=0.0034)$. The association between the mRNA expression of SENP2 and the clinicopathological characteristics including sex, age, stage, LDH expression and cell genetics of the 26/43 patients with CLL before treatment was also evaluated. As shown in Table II, the mRNA level of SENP2 was associated with the expression of LDH $(\mathrm{P}<0.05)$; however, no significant association was observed with the other clinicopathological characteristics examined.

Establishment of CLL cells in which SENP2 was overexpressed or silenced. To further explore the role of SENP2 in CLL cells in vitro, cell models in which SENP2 was overexpressed or silenced were established based on the human CLL cell line, MEC2. The overexpression and silencing of SENP2 were verified by western blot analysis and the results are shown in Fig. 1E. The results demonstrated that the expression of SENP2 was upregulated and downregulated in the cells in which SENP2 was overexpressed or silenced by shRNA, respectively.

Overexpression of SENP2 has the tendency to enhance the sensitivity of CLL cells to cytarabine and dexamethasone at an early stage. Cytarabine and dexamethasone are anticancer drugs commonly used in the treatment of leukemia. Thus, in this study, we examined the sensitivity of CLL cells, in which SENP2 was overexpressed or silenced, to chemotherapy, as this is also an important factor for determining the role of SENP2 in cancer. ACCK-8 assay was conducted to detect the response of the CLL cells, in which SENP2 was overexpressed
Table II. Association between the mRNA expression of SENP2 and the clinical or biological characteristics of the 26 patients CLL (untreated).

\begin{tabular}{lll}
\hline Characteristic & Median of $2^{-\Delta \text { cc }}($ range $)$ & P-value \\
\hline Sex & & \\
Male & $1.90(1.07-12.94)$ & 0.535 \\
Female & $2.46(1.01-5.06)$ & \\
Age (years) & & \\
$\geq 60$ & $2.21(1.07-12.94)$ & 0.607 \\
$<60$ & $1.93(1.01-8.10)$ & \\
Binet staging & & 0.382 \\
A and B & $1.84(1.07-8.10)$ & \\
C & $2.81(1.01-12.94)$ & \\
LDH expression & & \\
$>250 U / 1$ & $3.32(1.97-8.10)$ & \\
$\leq 250 U / 1$ & $1.74(1.01-12.94)$ & \\
$\beta 2-M G$ & & \\
$>3$ mg/l & $1.97(1.21-8.10)$ & \\
$\leq 3$ mg/l & $1.65(1.01-3.40)$ & \\
Cell genetics & & \\
With a poor & $2.46(1.52-4.19)$ & \\
prognosis index & & \\
Without a poor & $1.77(1.01-5.06)$ & \\
prognosis index & & \\
\hline
\end{tabular}



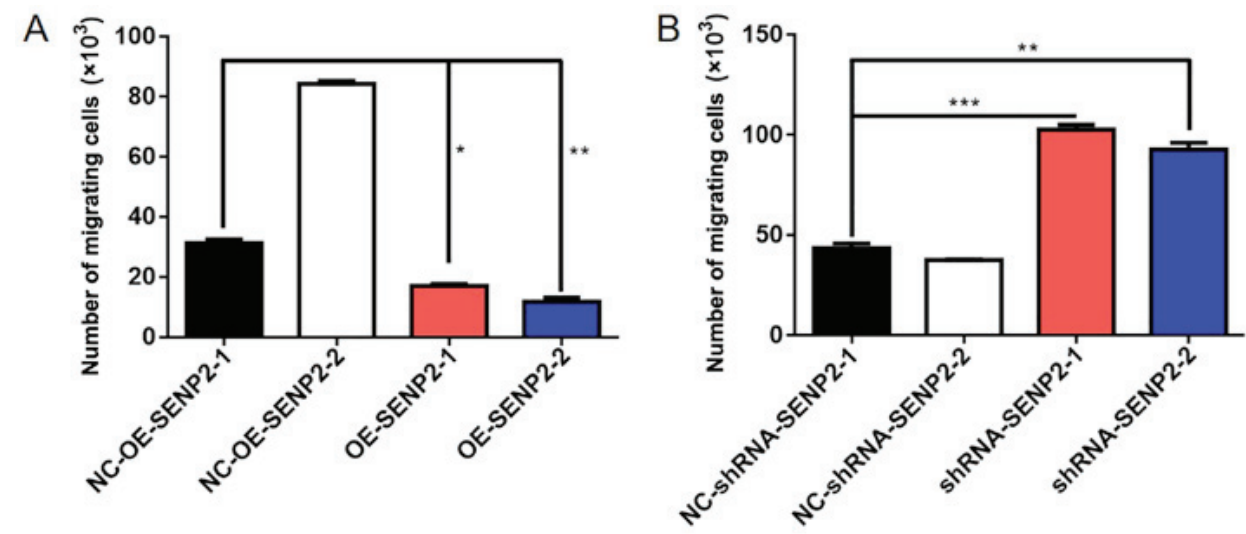

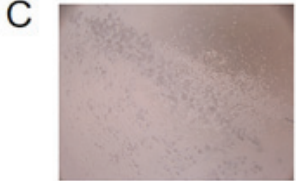

NC-OE-SENP2-1

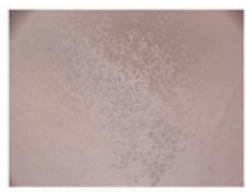

OE-SENP2-1

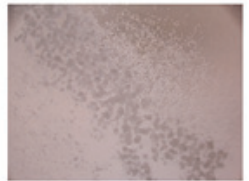

NC-OE-SENP2-2

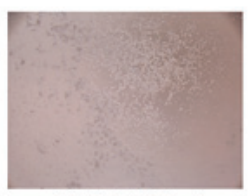

OE-SENP2-2
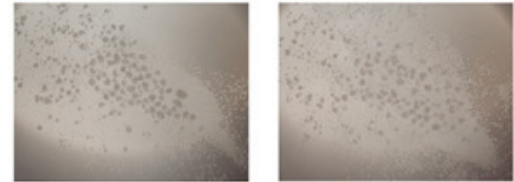

NC-shRNA-SENP2-1 NC-ShRNA-SENP2-2

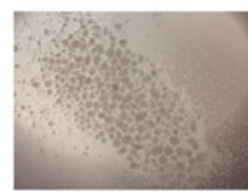

ShRNA-SENP2-1

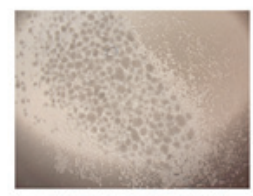

ShRNA-SENP2-2

Figure 3. Overexpression of SENP2 decreases the invasive ability of CLL cells. MEC2 cells in which SENP2 was (A and C) overexpressed or (B and D) silenced with their respective control cells were seeded into 6-well plates and they were then suspended in fresh medium without FBS after reaching to the 80-90\% confluence. Subsequently, a Transwell assay was conducted to determine the invasive ability of these cells. The statistical method used was a paired samples t-test. $\mathrm{P}<0.05$, ${ }^{* *} \mathrm{P}<0.01$ and ${ }^{* * *} \mathrm{P}<0.001$. CLL, chronic lymphocytic leukemia; NC-OE-SENP2, MEC2 cells transfected with null (control) overexpression vector; OE-SENP2, MEC2 cells transfected with SENP2 overexpression vector; NC-shRNA-SENP2, MEC2 cells transfected with null control shRNA; shRNA-SENP2, MEC2 cells transfected with shRNA against SENP2.

or silenced, to cytarabine or dexamethasone. As shown in Fig. 2, with the overexpression of SENP2, we observed a trend towards an increased the sensitivity of CLL cells to cytarabine and dexamethasone compared with the controls. The silencing of SENP2 was associated with a tendency towards increased drug resistance. However, with the prolongation of the treatment time with cytarabine and dexamethasone, the difference in the proliferation of the CLL cells in which SENP2 was overexpressed or silenced and the control group became inconspicuous. Collectively, it was hypothesized that SENP2 may enhance the sensitivity of CLL cells to chemotherapy at an early stage.

Overexpression of SENP2 decreases the invasive and chemotactic ability of CLL cells. Metastasis and invasion are crucial factors for grading malignancy in cancers; thus, we considered it essential to examine the effect of SENP2 on the invasive ability of CLL cells. Transwell assays were performed to examine the effect of SENP2 overexpression or silencing on the invasive ability of CLL cells and the results demonstrated that SENP2 overexpression resulted in fewer cells migrating from the upper chamber to the lower chamber, while the silencing of SENP2 in CLL cells had the opposite effect $(\mathrm{P}<0.01$ and $\mathrm{P}<0.001$, Fig. 3$)$.

In addition, the chemotactic response of leukocytes may reflect the prognosis for CLL patients; thus, we also examined the effect of SENP2 on the response of CLL cells to CXCL12 in a chemotaxis chamber. The data from the chemotaxis chamber assay revealed that the overexpression of SENP2 in the CLL cells inhibited the migration of the MEC 2 cells into the lower chamber (Fig. 4). When SENP2 was silenced, the CLL cells exhibited an increased chemotactic response. The increased expression of SENP2 decreased both the migration and chemotactic ability of the CLL cells.

Overexpression of SENP2 promotes the apoptosis of CLL cells. To investigate whether SENP2 promotes the apoptosis of CLL cells, western blot analysis and flow cytometry were used to detect the apoptosis rate of the CLL cells in which SENP2 was overexpressed following treatment with cytarabine or dexamethasone. The results of flow cytometry are shown in Fig. 5. It was found that following treatment with dexamethasone, the SENP2-overexpressing MEC2 cells exhibited more late-stage apoptosis than the control MEC2 cells. These results prove that SENP2 not only promotes the apoptosis of CLL cells, but also the ability of SENP2 to enhance the sensitivity of the cells to drug treatment with dexamethasone. When the SENP2-overexpressing MEC2 cells were treated with dexamethasone for 24,48 or $72 \mathrm{~h}$, the cell apoptosis increased in a time-dependent manner, and the cells also exhibited a time-dependent sensitivity to dexamethasone (Fig. 5).

In addition, SENP2 overexpression and treatment with dexamethasone or cytarabine appeared to increase the expression of the pro-apoptotic proteins, p53 compared with NC-OE-SENP2 but without statistical significance. Furthermore, 
A

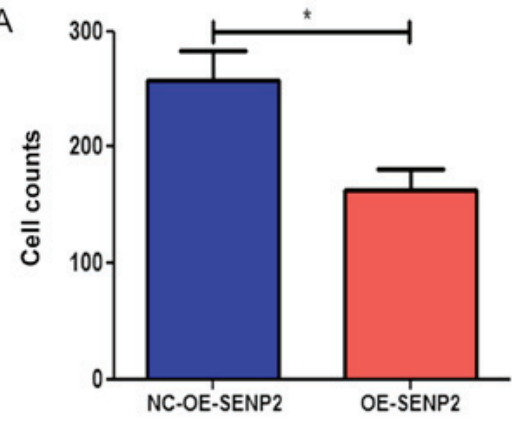

C NC-OE-SENP2

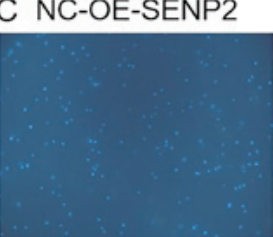

OE-SENP2

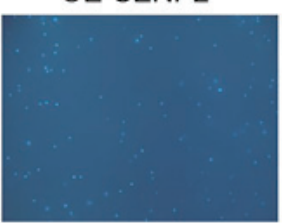

B

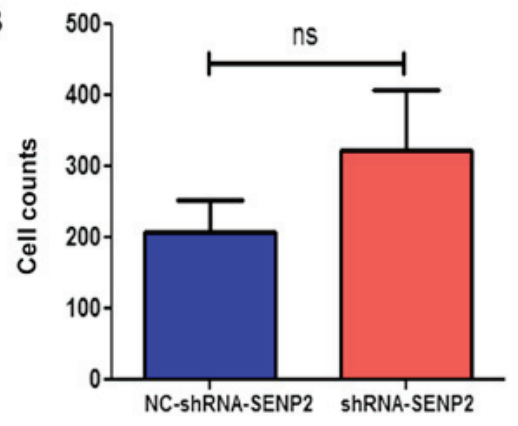

D NC-shRNA-SENP2

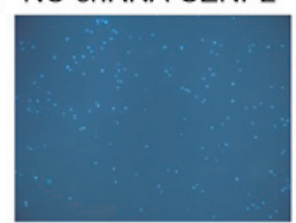

ShRNA-SENP2

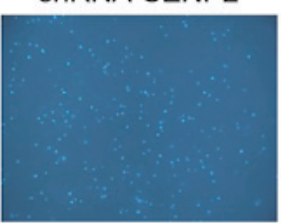

Figure 4. Overexpression of SENP2 enhances the chemotactic ability of CLL cells. MEC2 cells in which SENP2 was (A and C) overexpressed or (B and D) silenced with their respective control cells were treated as described in the Materials and methods and a chemotaxis chamber assay was then performed to inspect the chemotactic response of these cells. The statistical method used was a paired samples t-test. ${ }^{*} \mathrm{P}<0.05 ;$ ns, no statistical significance. CLL, chronic lymphocytic leukemia; NC-OE-SENP2, MEC2 cells transfected with null (control) overexpression vector; OE-SENP2, MEC2 cells transfected with SENP2 overexpression vector; NC-shRNA-SENP2, MEC2 cells transfected with null control shRNA; shRNA-SENP2, MEC2 cells transfected with shRNA against SENP2.
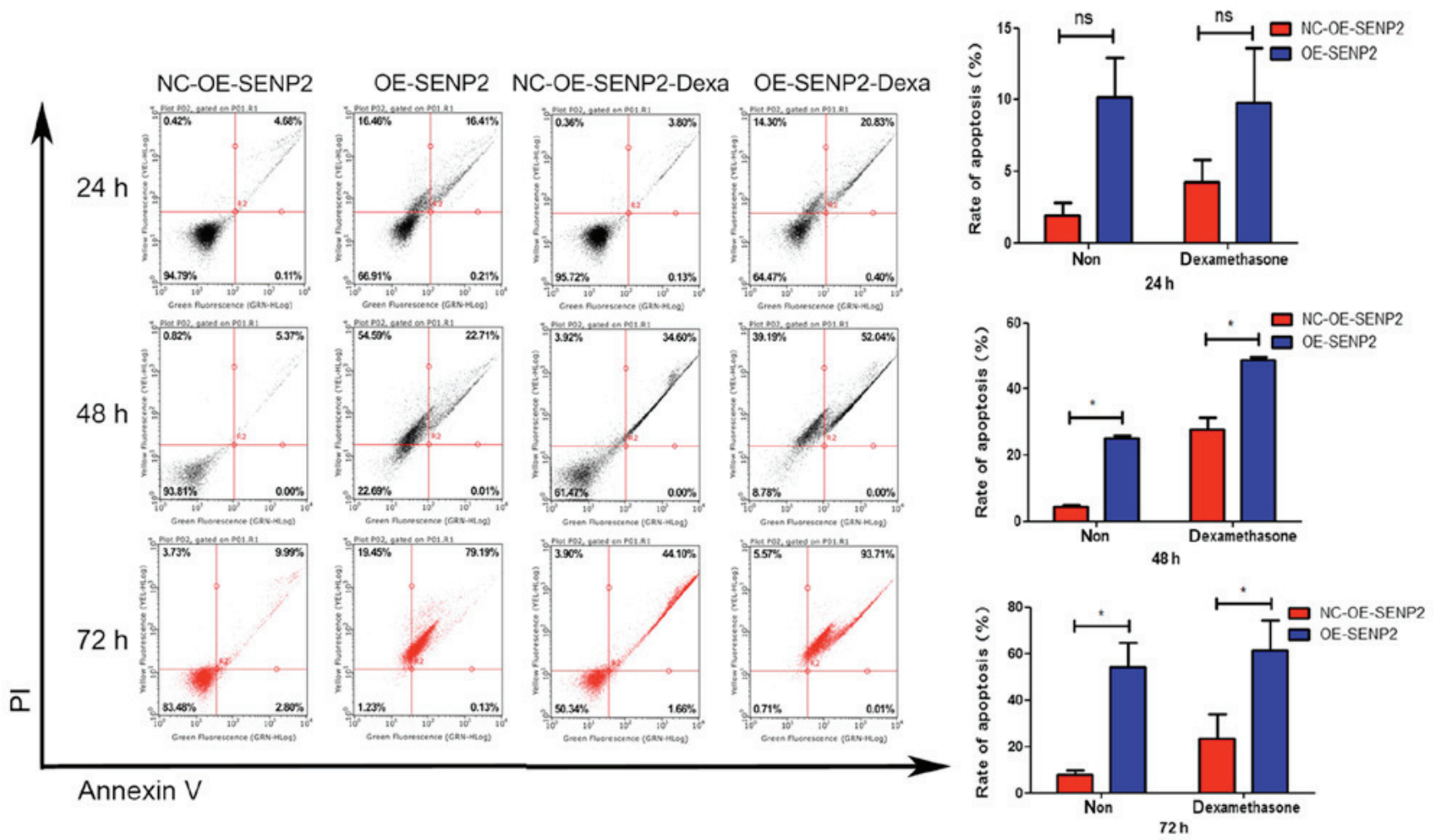

Figure 5. Overexpression of SENP2 promotes the apoptosis of CLL cells. The CLL cells in which SENP2 was overexpressed or silenced were seeded into $6 \mathrm{~cm}$ dishes. Upon reaching 60\% confluence, dexamethasone or DMSO were used to treat the CLL cells the cells were then further cultured for 24,48 or $72 \mathrm{~h}$. Subsequently, an Annexin V-FITC Apoptosis Detection kit was used to examine cell apoptosis. Finally, the cells were analyzed by flow cytometry following the addition of binding buffer $(400 \mu \mathrm{l})$. The statistical method used was a paired samples t-test. " $\mathrm{P}<0.05 ;$ ns, no statistical significance. CLL, chronic lymphocytic leukemia; NC-OE-SENP2, MEC2 cells transfected with null (control) overexpression vector; OE-SENP2, MEC2 cells transfected with SENP2 overexpression vector; NC-shRNA-SENP2, MEC2 cells transfected with null control shRNA; shRNA-SENP2, MEC2 cells transfected with shRNA against SENP2. Dexa, dexamethasone.

SENP2 overexpression and treatment with dexamethasone or cytarabine did not significantly affect the expression of other pro-apoptotic proteins, such as cleaved caspase-3, cleaved caspase- 9 and Bax, and on the expression of the anti-apoptotic protein, Bcl-2, compared with NC-OE-SENP2 (Figs. 6 and 7). The above-mentioned results indicate that SENP2 can increase 
A

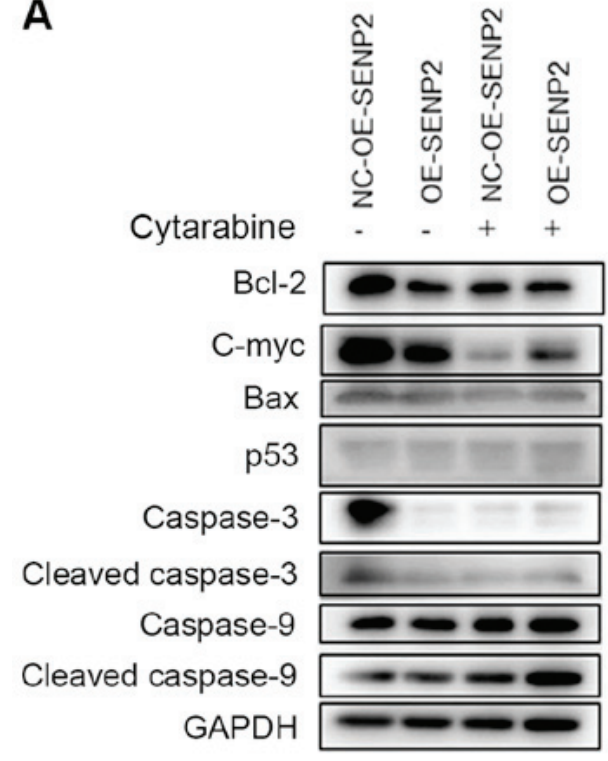

B

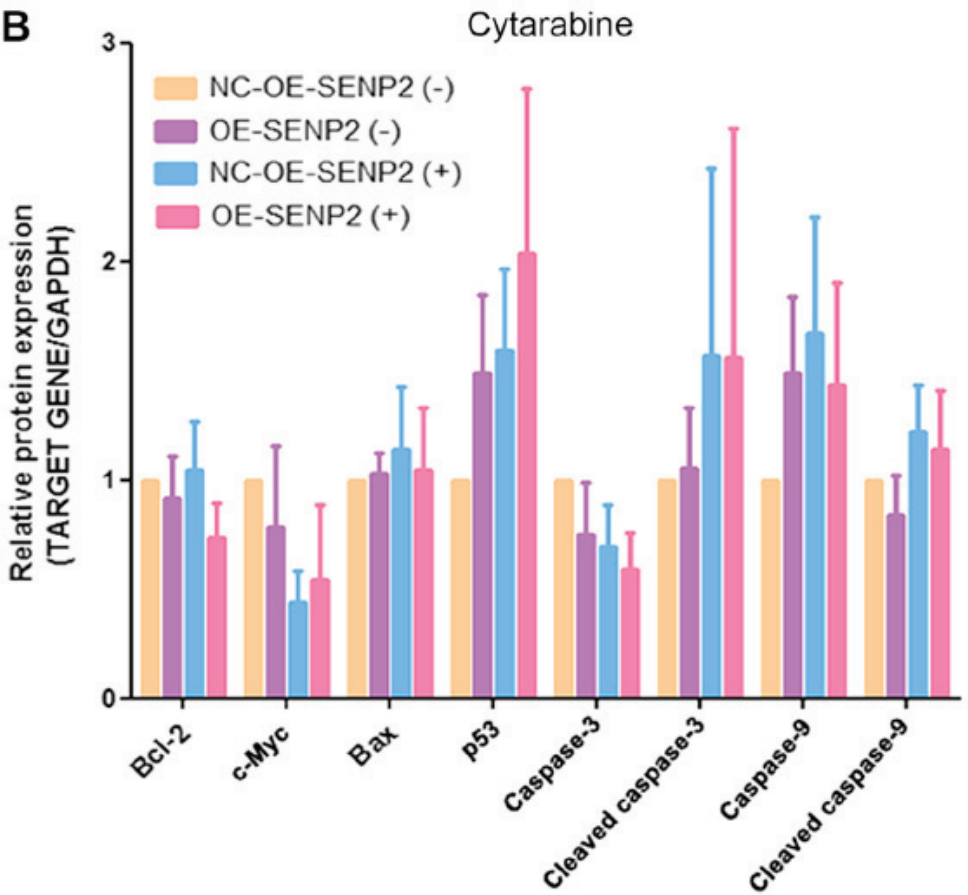

TARGET GENE

Figure 6. Effect of SENP2 overexpression on apoptosis-related proteins in CCL cells treated with or without cytarabine. (A and B) MEC2 cells in whichSENP2 was overexpressed and the control cells were cultured in $6 \mathrm{~cm}$ dishes and then treated with cytarabine for $24 \mathrm{~h}$ after reaching $70 \%$ confluence. Western blot analysis was then performed to inspect the expression levels of cleaved caspase-9, caspase-9, cleaved caspase-3, caspase-3, c-Myc, p53, Bax, and Bcl-2 in these cells. GAPDH was used to confirm equal amount of proteins loaded in each lane. CLL, chronic lymphocytic leukemia; NC-OE-SENP2, MEC2 cells transfected with null (control) overexpression vector; OE-SENP2, MEC2 cells transfected with SENP2 overexpression vector; NC-shRNA-SENP2, MEC2 cells transfected with null control shRNA; shRNA-SENP2, MEC2 cells transfected with shRNA against SENP2; +, cytarabine treatment, -, no cytarabine treatment.

A

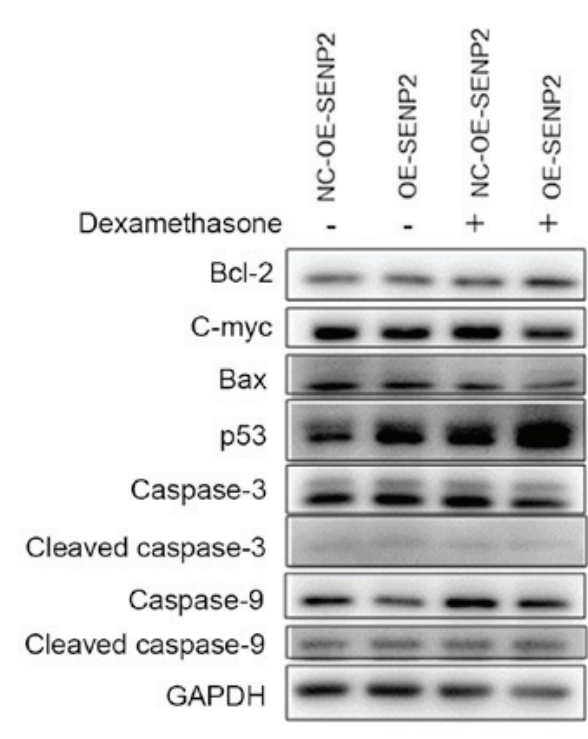

B

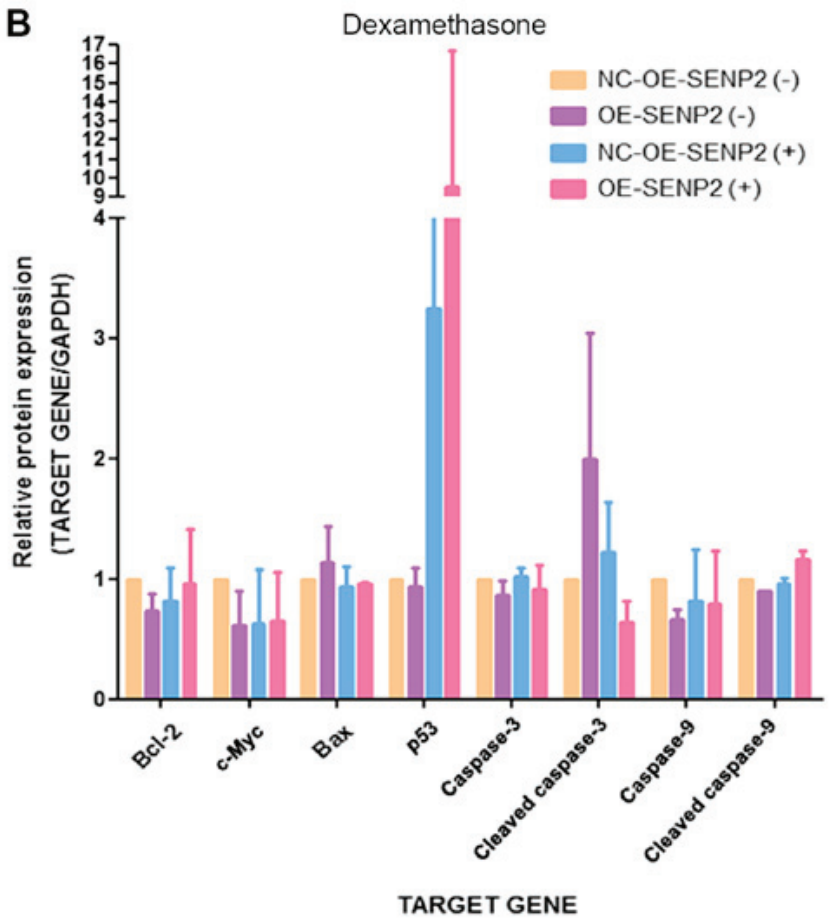

Figure 7. Effect of SENP2 overexpression on apoptosis-related proteins in CLL cells with or without dexamethasone treatment. (A and B) MEC2 cells in which SENP2 was overexpressed and the control cells were cultured in $6 \mathrm{~cm}$ dishes and then treated with dexamethasone for $24 \mathrm{~h}$ after reaching $70 \%$ confluence. Western blot analysis was then performed to inspect the expression levels of cleaved caspase- 9 , caspase- 9 , cleaved caspase-3, caspase-3, c-Myc, p53, Bax and Bcl-2 in these cells. GAPDH was used to confirm equal amount of proteins loaded in each lane. CLL, chronic lymphocytic leukemia; NC-OE-SENP2, MEC2 cells transfected with null (control) overexpression vector; OE-SENP2, MEC2 cells transfected with SENP2 overexpression vector; NC-shRNA-SENP2, MEC2 cells transfected with null control shRNA; shRNA-SENP2, MEC2 cells transfected with shRNA against SENP2; +, dexamethasone treatment, -, no dexamethasone treatment. 
A

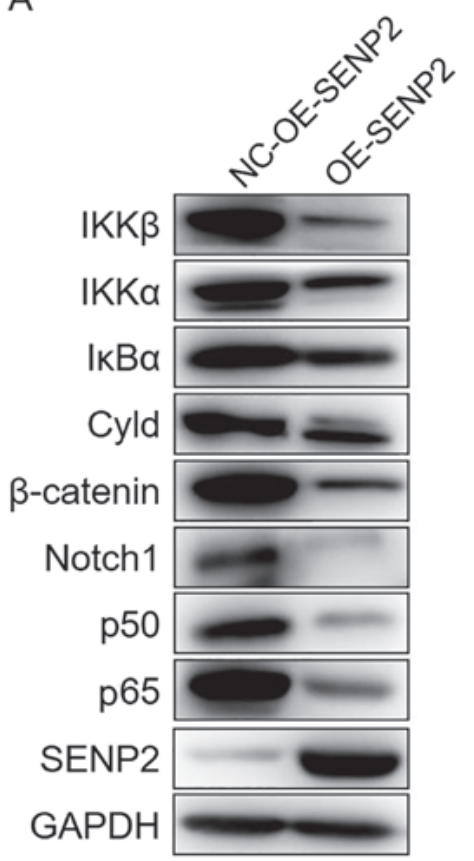

B

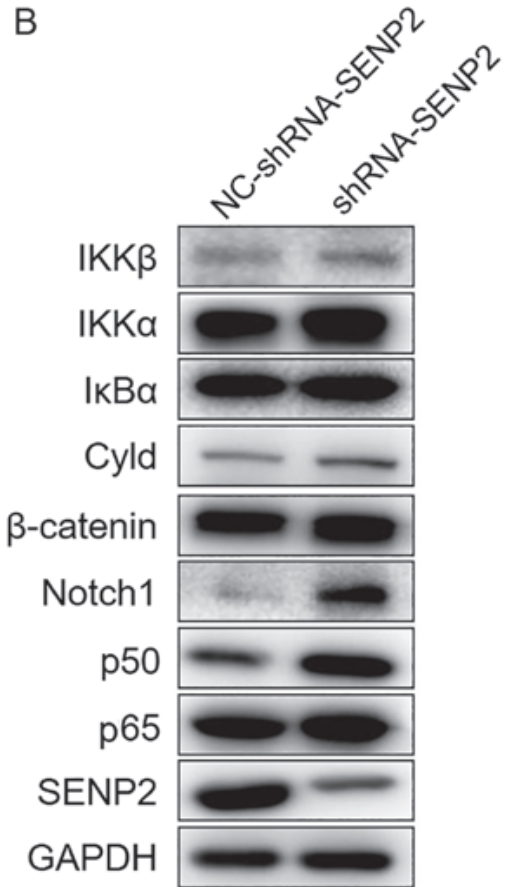

Figure 8. SENP2 suppresses the Notch and NF- $\kappa$ B signaling pathways. (A) SENP2-overexpressingMEC2 cells and the control cells were cultured in 6 cm dishes and they were harvested after reaching 80-90\% confluence, and these cells were then examind by western blot analysis to detect the expression levels of IKK $\beta$, IKK $\alpha$, I $\kappa \mathrm{B} \alpha$, Cyld, C-myc, $\beta$-catenin, Notch1, p50, p65 and SENP2. (B) MEC2 cells in which SENP2 was silenced and the control cells were treated as described in (A). GAPDH was used to confirm equal amount of proteins loaded in each lane. NC-OE-SENP2, MEC2 cells transfected with null (control) overexpression vector; OE-SENP2, MEC2 cells transfected with SENP2 overexpression vector; NC-shRNA-SENP2, MEC2 cells transfected with null control shRNA; shRNA-SENP2, MEC2 cells transfected with shRNA against SENP2.

the apoptosis of CLL cells in response to chemotherapeutics; however, the mechanisms involved warrant further investigation.

SENP2 suppresses the Notch and $N F-\kappa B$ signaling pathways. A number of studies have reported that the Notch signaling pathway is aberrantly activated in CLL cells and the activation of the Notch and NF- $\mathrm{B}$ signaling pathway can increase the survival of CLL cells $(17,18,20,22,24,25)$. Therefore, in order to further illustrate the molecular mechanisms of action of SENP2 in CLL cells, the expression of the Notch and NF- $\kappa B$ signaling pathways in CLL cells in which SENP2 was overexpressed or silenced was evaluated. As shown in Fig. 8A, the overexpression of SENP2 downregulated the expression of Cyld, IKK $\beta$, $\mathrm{I} \kappa \mathrm{B} \alpha, \mathrm{IKK} \alpha, \mathrm{p} 65$ and $\mathrm{p} 50$, all of which are proteins in the NF- $\kappa \mathrm{B}$ signaling pathway. The overexpression of SENP2 also downregulated the expression of Notch1 in the Notch signaling pathway. It has also been reported that SENP2 can regulate the stability of $\beta$-catenin (14). In this study, the MEC2 cells in which SENP2 was overexpressed also exhibited a downregulation in the expression of $\beta$-catenin (Fig. 8A). However, the silencing of SENP2 upregulated both the Notch signaling pathway and the NF- $\kappa \mathrm{B}$ signaling pathway (Fig. $8 \mathrm{~B}$ ). Based on these results, we can infer that SENP2 acts as a tumor suppressor in CLL cells through the regulation of the expression of $\beta$-catenin to suppress the Notch and NF- $\mathrm{B}$ signaling pathways.

\section{Discussion}

Recently, SENP2 was identified as a tumor suppressor in hepatocellular carcinoma, bladder cancer and breast cancer.
It has been shown that SENP2 can inhibit hepatocellular carcinoma cell growth by regulating the stability of WWOX that is essential for $\beta$-catenin stability $(14,15)$. In addition, the invasion and migration of bladder cancer cells has also been shown to be suppressed by SENP2 through the blocking of MMP13 expression (13). In 2014, Nait Achour et al reported that SENP2 inhibited the proliferation of breast cancer cells via the suppression of the transcription of estrogen receptor $\alpha(E R \alpha)$ signaling (12). Therefore, we wished to determine whether the anti-tumor effect of SENP2 can be applied to other malignant tumors and whether SENP2 may be a potential target for cancer treatment if the underlying mechanisms are determined. In this study, we selected a CLL model, which is the main subject of our research group, as the study object to explore the potential anti-tumor effects of SENP2 on CLL.

To date, at least to the best of our knowledge, there are no reports of the role of SENP2 in CLL. Therefore, we wished to determine whether the SENP2 is associated with the occurrence and development of CLL in situ. The peripheral blood of 43 patients with CLL and 21 healthy volunteers was collected to analyze the protein expression level of SENP2, and the results revealed that SENP2 was generally downregulated in the peripheral blood of patients with CLL with or without treatment compared with the healthy volunteers, although there was no statistically significant difference. As the sample size used for western blot analysis was limited, it would affect the results of statistical analysis which are prone to a type II error, leading to false-negative results. However, among the 4 patients before treatment and 3 patients after treatment who were randomly selected, 3 and 2 patients exhibited a 
significantly lower SENP2 expression compared to the control group in our western blot analysis. Thus, we explored the role of SENP2 in MEC2 cells using different experiments, although no statistically significant difference was observed between the healthy controls and patients with CLL. The differential mRNA expression levels of SENP2 in the patients with CLL and healthy volunteers revealed that the mRNA expression level of SENP2 in the patients with CLL was higher than that in both the healthy volunteers and post-treatment patients, indicating the differential expression of SENP2 in CLL cells which may be associated with chemotherapy. Notably, the results of western blot analysis and RT-qPCR appear to be contradictory, which may be attributed to the inconsistency between the transcriptional level and the protein expression level caused by the complex processes from mRNA translation to the formation of functional proteins.

It is well known that a poor prognosis of patients with CLL is associated with a greater age and later disease stage (1). Moreover, $\mathrm{LDH}, \beta 2-\mathrm{MG}$ and cytogenetic abnormalities are also commonly used factors for evaluating the prognosis of patients with CLL (28-31). Therefore, in this study, the association between the mRNA expression level of SENP2 and the clinicopathological characteristics of patients with CLL before treatment were analyzed for the first time, at least to the best of our knowledge. Among the variety of clinicopathological characteristics of CLL, the expression of LDH was demonstrated to be associated with the mRNA expression level of SENP2, indicating the possible involvement of SENP2 in the clinical course of disease. Considering that the number of samples in this study is small and the collected samples are mostly from inpatients and that we did not have early CLL specimens, the association between SENP2 and the prognostic factors of CLL warrants further investigation.

Since the aforementioned results provide the preliminary proofs of the potential involvement of SENP2 in CLL, whether SENP2 can affect the development of CLL needs to be determined. For this purpose, in this study, CLL cell models in which SENP2 was overexpressed or silenced were established to determine the effect of the expression level of SENP2 on the sensitivity of the cells to chemotherapy, and on cell viability, cell invasion and the apoptotic state following treatment with cytarabine and dexamethasone. Furthermore, the association between the expression of SENP2 and the activation state of the Notch and NF- $\kappa \mathrm{B}$ signaling pathways was also investigated to explore the underlying mechanisms. The successful construction of CLL cell lines in which SENP2 was overexpressed or silenced was verified by western blot analysis. Subsequently, the trend of chemosensitivity shown in Fig. 2 is consistent with our hypothesis that SENP2 enhances the sensitivity of MEC 2 cells to cytarabine and dexamethasone, although without statistical significance. Thus, we may need to further improve our experimental methods. At the same time, we noted that when the cells were treated with cytarabine or dexamethasone for $24 \mathrm{~h}$, obvious differences between the groups were observed, but at 48 and $72 \mathrm{~h}$, these differences were not obvious. It can thus be hypothesized that the overexpression of SENP2 has the tendency to enhance the sensitivity of CLL cells to cytarabine and dexamethasone at an early stage. However, as the time of drug action increases, tumor cells can antagonize this effect through other mechanisms. The mechanism of tumor drug resistance has been an important issue for researchers, which will also be the focus of our future research. Moreover, the results of Transwell and chemotaxis chamber assay in this study demonstrated that the overexpression of SENP2 decreased cell invasion and the cell chemotactic response, whereas the silencing of SENP2 led to the opposite results. Cell invasion reflects the invasive potential of cancer cells. These results also proved that SENP2 may exert anti-tumor effects on CLL cells by disrupting their chemotactic ability. In addition, the overexpression of SENP2 combined with cytarabine or dexamethasone treatment synergistically decreased the viability and the promoted apoptosis of CLL cells. In conclusion, these results completely verified that SENP2 exerts an anti-tumor effect on CLL by suppressing cell invasion and chemotactic ability, and inducing cell apoptosis and enhancing the sensitivity of CLL cells to chemotherapy.

Although we had decided that the anti-tumor effect of SENP2 could be applied to CLL from various routes and that the Notch signaling pathway may be involved, the underlying molecular mechanisms needed to be more fully examined. In our previous study, we reported that the Notch signaling pathway regulated the $\mathrm{NF}-\kappa \mathrm{B}$ signaling pathway in CLL cells, by disrupting the expression of RelA and RelB, key members in the NF- $\kappa \mathrm{B}$ family (24). Therefore, we inferred that the NF- $\kappa \mathrm{B}$ signaling pathway may also be involved in the regulation of SENP2 in CLL cells. In this study, western blot analysis was performed to analyze both the NF- $\kappa \mathrm{B}$ and Notch signaling pathway in the CLL cells in which SENP2 was overexpressed or silenced. The final results revealed that the overexpression of SENP2 simultaneously suppressed the NF- $\kappa \mathrm{B}$ and Notch signaling pathways, which may be a result of the reversible de-SUMOylation of proteins by SENP2. Notably, the silencing of SENP2 upregulated the Notch signaling pathway and NF- $\kappa \mathrm{B}$ signaling pathways. The study by Lee et al (32) suggested that the SUMO modification of the key molecule NEMO of the NF- $\mathrm{NB}$ signaling pathway is the key step for the activation of the NF- $\mathrm{B}$ signaling pathway following DNA damage. Therefore, in CLL, SENP2 may inhibit the activity of the NF- $\kappa \mathrm{B}$ signaling pathway by NEMO SUMO modification, while the activity of the NF- $\kappa \mathrm{B}$ signaling pathway reduces the expression of I $\mathrm{B}$. Based on these results, we summarized that the Notch signaling pathway and $N F-\kappa B$ signaling pathway may be regulated by SENP2. To explain this phenomenon, we inferred that there must be some other regulatory mechanisms between the SENP2 and the NF- $\mathrm{B}$ signaling pathway which need to be investigated in future studies.

Collectively, this study first explored the expression of SENP2 in primary CLL cells and the association between SENP2 and CLL-related prognostic factors. We further examined the effect of SENP2 on CLL cell functions and the effect of SENP2 on key signaling pathways in CLL, including the Notch, Wnt/ $\beta$-catenin and NF- $\kappa \mathrm{B}$ pathways. However, the number of CLL cases collected in this study was relatively small, and thus some of the results may not be convincing. In addition, we did not further explore the mechanisms of action of SENP2 and its association with SUMOylation and de-SUMOylation. Therefore, in future studies, we aim to expand the sample size to further clarify the association between SENP2 and CLL prognosis, as well as with chemotherapy, as well as to illustrate the functional location of 
SENP2, and to explore the mechanisms of SENP2 as regards the regulation of the Notch, Wnt/ $\beta$-catenin, and NF- $\kappa \mathrm{B}$ signaling pathways and its association with SUMOylation and de-SUMOylation.

In conclusion, the results of this study clearly illustrate that SENP2 expression is generally downregulated in patients with CLL and may inhibit cell invasion and the cell chemotactic ability, and promote cell chemosensitivity and cell apoptosis in CLL by suppressing the NF- $\mathrm{BB}$ and Notch signaling pathways. This study may provide a theoretical basis for the development of novel therapeutic strategies for CLL treatment.

\section{Acknowledgements}

Not applicable.

\section{Funding}

This study was supported by the Natural Science Foundation of Fujian Province of China (No. 2016J01458), Joint Funds for the Innovation of Science and Technology of Fujian Province of China (No. 2017Y9005), the Construction Project of Fujian Medical Center of Hematology (Min201704), and the National and Fujian Provincial Key Clinical Specialty Discipline Construction Program, P.R. China.

\section{Availability of data and materials}

All data generated or analyzed during this study are included in this published article. The datasets used and/or analyzed during the current study are available from the corresponding author on reasonable request.

\section{Authors' contributions}

XLC, SFW and ZSX conceived and designed and supervised the study; XLC, SFW, XTL, HXL and TTW performed the experiments; SQW, ZJQ and RZ analyzed the data; XLC, SFW, RZ and ZSX wrote the manuscript. All authors have read and approved the final manuscript.

\section{Ethics approval and consent to participate}

The use of human samples in this study was approved by the Ethics Committee of the Fujian Medical University Union Hospital and written consent was obtained from all participants in this study.

\section{Patient consent for publication}

Not applicable.

\section{Competing interests}

The authors declare that they have no competing interests.

\section{References}

1. Nabhan C and Rosen ST: Chronic lymphocytic leukemia: A clinical review. JAMA 312: 2265-2276, 2014
2. Barrientos JC: Management of chronic lymphocytic leukemia in the elderly. Cancer Contr 22 (Suppl): S17-S23, 2015.

3. Stilgenbauer S, Furman RR and Zent CS: Management of chronic lymphocytic leukemia. Am Soc Clin Oncol Educ Book 35: 164-175, 2015.

4. Xu Z, Zhang J, Wu S, Zheng Z, Chen Z and Zhan R: Younger patients with chronic lymphocytic leukemia benefit from rituximab treatment: A single center study in China. Oncol Lett 5: 1266-1272, 2013.

5. Jamroziak K, Puła B and Walewski J: Current treatment of chronic lymphocytic leukemia. Curr Treat Options Oncol 18: 5, 2017.

6. Chow KH, Elgort S, Dasso M, Powers MA and Ullman KS: The SUMO proteases SENP1 and SENP2 play a critical role in nucleoporin homeostasis and nuclear pore complex function. Mol Biol Cell 25: 160-168, 2014.

7. Goeres J, Chan PK, Mukhopadhyay D, Zhang H, Raught B and Matunis MJ: The SUMO-specific isopeptidase SENP2 associates dynamically with nuclear pore complexes through interactions with karyopherins and the Nup107-160 nucleoporin subcomplex. Mol Biol Cell 22: 4868-4882, 2011.

8. Kim JH and Baek SH: Emerging roles of desumoylating enzymes. Biochim Biophys Acta 1792: 155-162, 2009.

9. Kang X, Qi Y, Zuo Y, Wang Q, Zou Y, Schwartz RJ, Cheng J and Yeh ET: SUMO-specific protease 2 is essential for suppression of polycomb group protein-mediated gene silencing during embryonic development. Mol Cell 38: 191-201, 2010.

10. Jiang M, Chiu SY and Hsu W: SUMO-specific protease 2 in Mdm2-mediated regulation of p53. Cell Death Differ 18: 1005-1015, 2011.

11. Chiu SY, Asai N, Costantini F and Hsu W: SUMO-specific protease 2 is essential for modulating p53-Mdm2 in development of trophoblast stem cell niches and lineages. PLoS Biol 6: e310, 2008.

12. Nait Achour T, Sentis S, Teyssier C, Philippat A, Lucas A, Corbo L, Cavaillès V and Jalaguier S: Transcriptional repression of estrogen receptor $\alpha$ signaling by SENP2 in breast cancer cells. Mol Endocrinol 28: 183-196, 2014.

13. Tan M, Gong H, Wang J, Tao L, Xu D, Bao E, Liu Z and Qiu J: SENP2 regulates MMP13 expression in a bladder cancer cell line through SUMOylation of TBL1/TBLR1. Sci Rep 5: 13996, 2015.

14. Shen HJ, Zhu HY, Yang C and Ji F: SENP2 regulates hepatocellular carcinoma cell growth by modulating the stability of $\beta$-catenin. Asian Pac J Cancer Prev 13: 3583-3587, 2012.

15. Jiang QF, Tian YW, Shen Q, Xue HZ and Li K: SENP2 regulated the stability of $\beta$-catenin through WWOX in hepatocellular carcinoma cell. Tumour Biol 35: 9677-9682, 2014.

16. Artavanis-Tsakonas S, Rand MD and Lake RJ: Notch signaling: Cell fate control and signal integration in development. Science 284: 770-776, 1999.

17. Nwabo Kamdje AH, Bassi G, Pacelli L, Malpeli G, Amati E, Nichele I, Pizzolo $G$ and Krampera M: Role of stromal cell-mediated Notch signaling in CLL resistance to chemotherapy. Blood Cancer J 2: e73, 2012.

18. Rosati E, Sabatini R, Rampino G, Tabilio A, Di Ianni M, Fettucciari K, Bartoli A, Coaccioli S, Screpanti I and Marconi P: Constitutively activated Notch signaling is involved in survival and apoptosis resistance of B-CLL cells. Blood 113: 856-865, 2009.

19. Balatti V, Lerner S, Rizzotto L, Rassenti LZ, Bottoni A, Palamarchuk A, Cascione L, Alder H, Keating MJ, Kipps TJ, et al: Trisomy 12 CLLs progress through NOTCH1 mutations. Leukemia 27: 740-743, 2013.

20. Rossi D, Rasi S, Fabbri G, Spina V, Fangazio M, Forconi F, Marasca R, Laurenti L, Bruscaggin A, Cerri M, et al: Mutations of NOTCH1 are an independent predictor of survival in chronic lymphocytic leukemia. Blood 119: 521-529, 2012

21. Herishanu Y, Pérez-Galán P, Liu D, Biancotto A, Pittaluga S, Vire B, Gibellini F, Njuguna N, Lee E, Stennett L, et al: The lymph node microenvironment promotes B-cell receptor signaling, NF-kappaB activation, and tumor proliferation in chronic lymphocytic leukemia. Blood 117: 563-574, 2011.

22. Fabbri G, Rasi S, Rossi D, Trifonov V, Khiabanian H, Ma J, Grunn A, Fangazio M, Capello D, Monti S, et al: Analysis of the chronic lymphocytic leukemia coding genome: Role of NOTCH1 mutational activation. J Exp Med 208: 1389-1401, 2011.

23. Puente XS, Pinyol M, Quesada V, Conde L, Ordóñez GR, Villamor N, Escaramis G, Jares P, Beà S, González-Díaz M, et al: Whole-genome sequencing identifies recurrent mutations in chronic lymphocytic leukaemia. Nature 475: 101-105, 2011. 
24. Xu ZS, Zhang JS, Zhang JY, Wu SQ, Xiong DL, Chen HJ, Chen ZZ and Zhan R: Constitutive activation of NF- $\kappa$ B signaling by NOTCH1 mutations in chronic lymphocytic leukemia. Oncol Rep 33: 1609-1614, 2015

25. Baldoni S, Sportoletti P, Del Papa B, Aureli P, Dorillo E, Rosati E, Ciurnelli R, Marconi P, Falzetti F and Di Ianni M: NOTCH and $\mathrm{NF}-\kappa \mathrm{B}$ interplay in chronic lymphocytic leukemia is independent of genetic lesion. Int J Hematol 98: 153-157, 2013.

26. Sun Q, Wang R, Luo J, Wang P, Xiong S, Liu M and Cheng B Notch1 promotes hepatitis $B$ virus $X$ protein-induced hepatocarcinogenesis via $\mathrm{Wnt} / \beta$-catenin pathway. Int $\mathrm{J}$ Oncol 45 : $1638-1648,2014$

27. Livak KJ and Schmittgen TD: Analysis of relative gene expression data using real-time quantitative PCR and the 2(- $\Delta \Delta \mathrm{C}(\mathrm{T}))$ Method. Methods 25: 402-408, 2001.

28. Parikh SA and Shanafelt TD: Prognostic factors and risk stratification in chronic lymphocytic leukemia. Semin Oncol 43: 233-240, 2016
29. Farah R, Al Danaf J, Braiteh N, Costa JM, Farhat H, Mariani G and Giansily-Blaizot M: Life-threatening bleeding in factor VII deficiency: The role of prenatal diagnosis and primary prophylaxis. Br J Haematol 168: 452-455, 2015.

30. Zenz T, Mertens D, Küppers R, Döhner H and Stilgenbauer S: From pathogenesis to treatment of chronic lymphocytic leukaemia. Nat Rev Cancer 10: 37-50, 2010.

31. Seiffert M, Dietrich S, Jethwa A, Glimm H, Lichter P and Zenz T: Exploiting biological diversity and genomic aberrations in chronic lymphocytic leukemia. Leuk Lymphoma 53: 1023-1031, 2012.

32. Lee MH, Mabb AM, Gill GB, Yeh ET and Miyamoto S: NF- $\kappa B$ induction of the SUMO protease SENP2: A negative feedback loop to attenuate cell survival response to genotoxic stress. Mol Cell 43: 180-191, 2011.

This work is licensed under a Creative Commons Attribution-NonCommercial-NoDerivatives 4.0 International (CC BY-NC-ND 4.0) License. 